\title{
IMPROVED ANGIOGRAPHIC MEASUREMENT OF CORONARY ARTERY BLOOD FLOW
}

\author{
P.-A. Dorsaz, L. Dorsaz, P.-A. Doriot, W. Rutishauser \\ Cardiology Center, University Hospital, Geneva, Switzerland
}

INTRODUCTION: Absolute measurements of mean coronary blood flow in the cardiac catheterization laboratory by use of thermodilution or ultrasonic methods being very problematic, we have chosen, some years ago, to develop an angiographic approach yielding the mean left or right coronary flow in $\mathrm{ml} / \mathrm{min}$ according to the definition "mean flow = volume of fluid passing through the considered cross section divided by the corresponding time interval". Our present method is based on biplane cinefilm or digital recording of a sustained, constant rate injection of contrast medium into the proximal left or right coronary artery. The main coronary branches are then 3D-reconstructed and "concentration-distance curves" are computed and thresholded to yield the flow.

While in vitro experiments showed that the needed intravascular volumes can be obtained accurately [1], in vivo validation measurements [2] suggested, however, that some aspects were not sufficiently well understood. A computer simulation was therefore designed to study the propagation of contrast medium in a coronary artery [3]. It led to 2 main predictions: 1) The best appropriate threshold is about $50 \%$ of the concentration at the injection site. 2) Flow will usually be underestimated if the rate at which contrast medium is injected is less than peak coronary flow.

In order to verify these points in vivo, flow measurements were performed on patients by use of adequate and inadequate injection rates. In some patients, comparative measurements by means of an intracoronary Doppler device could also be performed. In this contribution, we present preliminary results of this experimental study.

METHOD: The 3D-reconstruction method has been described in detail elsewhere [1]. Its main steps are the following: after biplane cinefilm recording at 50 frames/s of an ECG-triggered power injection of lopamiro-370 into the proximal part of the left or right coronary artery, a $4 \mathrm{~cm}$ cube bearing 15 steel markers is filmed at the approximate place occupied previously by the patient's heart. The cube defines an arbitrary cartesian coordinate system in space, with respect to which the spatial coordinates of the 15 markers are precisely known. This is used subsequently to determine the projection parameters in form of two projection matrices.

The apparatus for cinefilm digitization is presently composed of a $35 \mathrm{~mm}$ cinefilm projector coupled to a CCD scanner driven by a Macintosh microcomputer. The images, digitized into a $M \times N \times 8$ bits matrix ( $M$ and $N$ can be chosen by the user), are then transferred to a workstation (Sun Sparc 2). They are processed therein by a dedicated program implemented inside OSIRIS, the general purpose medical imaging software developed by the Imaging Unit of our hospital [4]. Alternatively, images acquired readily in digital form ( 25 frames/s per plane) by the angiographic equipment (Bicor/Hicor/Awos, Siemens, [5]) can also be transferred into the workstation.

The two projection matrices are determined first from a pair of cineframes of the cube. The main coronary branches are then 3D-reconstructed from a pair of nearly simultaneous cineframes showing them fully opacified. This allows to compute the needed intravascular volumes. For the determination of absolute coronary flow in $\mathrm{ml} / \mathrm{min}$, the concentration of contrast medium along the branches is then computed (in arbitrary units) from two pairs of cineframes, the first one taken just after injection onset, and the second one exactly one cardiac cycle later (All three coronary image pairs are of course phasic). This yields a "concentrationdistance" curve per branch. The volume of fluid (contrast medium mixed with blood) which has flown into the arteries during the considered cardiac cycle is then obtained by thresholding the concentrationdistance curves appropriately.

In order to verify the predictions of the computer simulation about the appropriate concentration threshold $(50 \%)$ and the adequate injection rate, flow measurements were performed as follows on patients undergoing routine cardiac catheterization. After completion of the routine examination, the patient received (as a rule) two constant rate injections of either $3 \mathrm{ml} / \mathrm{s}$ and $6 \mathrm{ml} / \mathrm{s}$, or $3 \mathrm{ml} / \mathrm{s}$ and 1.5 $\mathrm{ml} / \mathrm{s}$. The mean coronary flow was then determined for each injection according to our method. Reviewing the cinefilms, the injections were noted "strong" if a permanent reflux into the aorta could be observed over the whole injection duration. Otherwise, the 
injection was noted "weak". This resulted in 6 pairs of the type "strong/strong" and 18 pairs of the types "strong/weak" or "weak/strong".

In the more recent patients, comparative flow measurements could be performed by means of a new intracoronary Doppler flow-velocity measuring system with very small probe diameter $(0.45 \mathrm{~mm}$; "FloWire", Cardiometrics). The probe was placed in the proximal part of the coronary artery. The mean flow was obtained by multiplying the halved average instantaneous peak velocity with the corresponding cross sectional vessel area.

RESULTS: The flow values obtained from two corresponding "strong" injections (6 pairs) were nearly equal (mean relative unsigned difference: $8 \%$; range: $2 \%$ to $11 \%$ ). This demonstrates that flow values obtained with injection rates equal to or greater than peak coronary flow are reproducible even with very different injection rates.

Comparison of the flow values obtained from the strong injections by use of our 3D method with the corresponding values obtained with the "FloWire" shows at present an average absolute difference of $25 \%$, with a standard deviation of $11 \%$ ( range: $-30 \%$ to $45 \%)$. The flow values obtained angiographically are so far in relatively good agreement with those of the ultrasonic system.

As predicted by the computer simulation, the "weak" injections resulted regularly in lower flow values than the corresponding "strong" injections, independently of the chronological order of the injections. The average flow underestimation obtained from the 18 pairs "strong/weak" or "weak/ strong" was $31 \%$ (range : $-63 \%$ to $-8 \%$ ). This confirms that too low injection rates result in flow underestimation.

DISCUSSION: Coronary blood flow measurement in the cardiac catheterization laboratory has always been a difficult problem. In the late ten years, many efforts were devoted to the improvement of methods for myocardial perfusion assessment, but these methods yield only relative flow values. Some earlier approaches were problematic for various reasons. For instance, angiographic approaches could not include 3D-reconstruction. Moreover, the strong pulsatile character of coronary flow had often to be ignored. Since recently, intracoronary Doppler velocity measurements were impaired by the flow reduction induced by the relatively large diameter of the ultrasonic probe.

Our method yields readily the mean coronary flow in $\mathrm{ml} / \mathrm{min}$. As with methods based on the indicator dilution theory, some basic assumptions about the properties of the indicator (contrast medium) are necessary. However, since the pulsatile character of coronary flow is readily taken into account, no special assumption is necessary in this respect. While 3D-reconstruction proved to be sufficiently accurate, the difficulty was to find out if an appropriate thresh- old value exists for the great variety of coronary flow pulses and velocity profiles. This question was answered theoretically by the results of our computer simulation. The present experimental results confirm the prediction about the importance of the injection rate. Indeed, flow values obtained from injection pairs of the type "strong/strong" were rather comparable, while flow values obtained from a "weak" injection were clearly lower than the corresponding values obtained from the "strong" injection.

The results confirm also the prediction about the appropriateness of a $50 \%$ threshold value in the sense that flow values obtained from a "strong" injection were rather comparable to the corresponding values obtained by use of the "FloWire" device. Taking into account that both kind of measurements could not be performed simultaneously, and also that Doppler measurements of this kind cannot be very accurate for various reasons (Difficult centering of the probe in the proximal part of the artery, non parabolic velocity profile, limited accuracy of the cross sectional area determination, etc), the agreement between angiographic and ultrasonic flow values is surprisingly good.

CONCLUSION: The preliminary results presented in this contribution show that: 1) our angiographic method yields relatively accurate flow values when the injection rate is at least equal to peak coronary flow, provided that the applied threshold is $50 \%$, and: 2) too low injection rates result usually in considerable flow underestimation.

\section{REFERENCES:}

[1] Guggenheim N., Doriot P.-A., Dorsaz P.-A., Descouts P., Rutishauser W.: Spatial reconstruction of coronary arteries from angiographic images. Phys Med Biol 36/1: 99-110, 1991.

[2] Guggenheim N., Dorsaz P.-A., Doriot P.-A., Suilen C., Chappuis F., Rutishauser W.: 3D determination of the intravascular volume and flow of coronary arteries. Int J Bio-Med Computing 35: 13-23, 1993

[3] Doriot P.-A., Guggenheim N., Dorsaz P.-A., Chappuis F., Rutishauser W.: Contrast medium concentration threshold for angiographic measurements of absolute coronary blood flow. In: Computers in Cardiology 1992, IEEE Computer Society: 183-186, 1992.

[4] Ligier Y., Ratib O., Logean M., Girard Ch., Perrier R., Scherrer J.-R.: Object-oriented design of medical imaging software. Computerized Medical Imaging and Graphics, 18/2: 125-135, 1994.

[5] Doriot P.-A., Urban Ph., Adatte J.J., Rutishauser W.: HICOR for digital biplane angiocardiography. Assessment after one year's clinical use. Electromedica 62/1: 14-18, 1994. 